\title{
MORPHOLOGICAL SIGNAL ADAPTIVE MEDIAN FILTER FOR STILL IMAGE AND IMAGE SEQUENCE FILTERING
}

\author{
Sofia Tsekeridou Constantine Kotropoulos Ioannis Pitas \\ Department of Informatics \\ Aristotle University of Thessaloniki \\ Box 451, 54006 Thessaloniki, GREECE. \\ Tel: +30-31-996304, Fax: +30-31-996304 \\ E-mail: pitas@zeus.csd.auth.gr
}

\begin{abstract}
A novel extension of the classical signal-adaptive median filter $(S A M)$ is proposed in this paper, namely the morphological signaladaptive median filter $(M S A M)$. Three modifications are introduced in the $S A M$ filter aiming at: (1) enhancing the $S A M$ impulse detection mechanism so that it also detects randomly-valued impulses, (2) employing an anisotropic window adaptation based on binary morphological erosions/dilations with predefined structuring sets and (3) extending its design for its application to image sequences as well. Its performance has been tested for noise suppression in both still images and image sequences. Its robustness against a wide variety of noise distributions as well as its superiority to the classical $S A M$ filter are proved judging from both objective ( $S N R, M A E$ ) and subjective (perceived image quality) criteria.
\end{abstract}

\section{INTRODUCTION}

A frequently encountered problem in image processing is the corruption of images or image sequences by a variety of noise distributions dependent on the prevalent conditions. The observed noise can be modeled either as additive white, impulsive, signaldependent or a combination of them [1]. Therefore, the need emerges for implementing smoothing techniques that are able to treat different kinds of noise. Furthermore, a noise-free version of the corrupted image or sequence required by adaptive filtering algorithms during the training procedure is not always available. Moreover, it is well known that the main objectives of image filtering algorithms are: (a) the suppression of noise in homogeneous regions, (b) the preservation of edges (spatial or temporal) and (c) the removal of impulses (of constant and/or random value). A class of filters that fulfills these requirements is the so called signaladaptive filters. Signal-adaptive median $(S A M)$ is a paradigm of this class [1].

A novel extension of the classical $S A M$ filter [1] is proposed in this paper, namely the morphological signal-adaptive median filter $(M S A M)$. This filter performs well on many kinds of noise. It does not require a priori knowledge of a noise-free image or frame, but only of certain noise characteristics, which can be easily estimated. It adapts its behaviour based on a local $S N R$ measure achieving thus edge preservation and noise smoothing in homogeneous regions. It smooths impulsive noise as well.

\section{MORPHOLOGICAL SIGNAL ADAPTIVE MEDIAN FILTER STRUCTURE}

To begin with, let us describe the framework for signal-adaptive filters. Let $\sigma_{n}^{2}$ denote the noise variance that is known or has been estimated beforehand. Moreover, in the case of impulsive noise let $p_{p}$ be the percentage of positive impulses (i.e., $S_{\max }=255$ ), and $p_{n}$ be the percentage of negative impulses (i.e., $S_{\min }=0$ ). Thus, the noisy image pixel values $x(k, l)$ are determined by the model:

$$
x(k, l)= \begin{cases}S_{\min } & \text { with prob. } p_{n} \\ S_{\max } & \text { with prob. } p_{p} \\ x(k, l) & \text { with prob. } 1-\left(p_{p}+p_{n}\right)\end{cases}
$$

where $x(k, l)$ is an image pixel possibly corrupted by additive white or signal-dependent noise. An image can be considered as a sum of two components:

$$
x(k, l)=x_{L}(k, l)+x_{H}(k, l)
$$

i.e., a low-frequency component $x_{L}(k, l)$ which is dominant in homogeneous regions, and a high frequency one $x_{H}(k, l)$ observed in edges. The output of the $M S A M$ filter is expressed as in the $S A M$ filter, i.e.

$$
y(k, l)=\tilde{x}_{M}(k, l)+b(k, l)\left[x(k, l)-\tilde{x}_{M}(k, l)\right]
$$

$\tilde{x}_{M}(k, l)$ is the modified median, i.e., the median of the pixels that remain after the removal of impulses from the local window. $b(k, l)$ is a weighting coefficient that is used to adapt the window size according to whether a flat region or an edge has been met. It is evident that total noise suppression is achieved in homogeneous regions, because a large window is employed due to $b(k, l)$ being close to 0 . Edges are also preserved well, because a small window size is used due to $b(k, l)$ being close to 1 in this case. The window increment/decrement procedure is explained below. Two major modifications are introduced in the $M S A M$ filter structure compared to the $S A M$ filter [1]:

(1) $M S A M$ employs binary dilations and erosions with certain predefined structuring elements (SEs) in order to vary the window size anisotropically with respect to the local image content.

(2) $M S A M$ employs two impulse detectors: one for constant impulses (either positive or negative) and another for randomly-valued impulses. Impulse detection is done only in the initial window of dimensions $3 \times 3$. 
Subsequently, the several steps of the algorithm are presented.

\section{Constant value impulse detection [1]:}

The filter performs detection of constant value impulses in an initial window of dimensions $3 \times 3$ by using a signal-dependent threshold for negative impulses, $\tau_{n}(k, l)$, and another one for positive impulses, $\tau_{p}(k, l)$,

$$
\begin{aligned}
\tau_{n}(k, l) & =c\left[S_{\min }-\tilde{x}_{M}(k, l)\right] \leq 0 \\
\tau_{p}(k, l) & =c\left[S_{\max }-\tilde{x}_{M}(k, l)\right] \geq 0
\end{aligned}
$$

where $\mathrm{c}$ is a constant equal to $5 / 6$. If $\left[x(k, l)-\tilde{x}_{M}(k, l)\right]<$ $\tau_{n}$ then $x(k, l)$ is detected as a negative impulse. Similarly, if $\left[x(k, l)-\tilde{x}_{M}(k, l)\right]>\tau_{p}$ then $x(k, l)$ is detected as a positive impulse.

\section{Randomly-valued impulse detection:}

Motivated by the randomly-valued impulse detection mechanisms developed in [3, 2], two additional thresholds are introduced in the $S A M$ filter, defined by:

$$
\begin{aligned}
& h_{1}(k, l)=x_{\max }-x_{\min 2} \\
& h_{2}(k, l)=x_{\max 2}-x_{\min }
\end{aligned}
$$

$x_{m i n}$ is the minimum value pixel, $x_{m i n 2}$ is the second minimum value pixel, $x_{\max }$ is the maximum value pixel and $x_{\max 2}$ is the second maximum value pixel in the initial window. If $\mid x(k, l)-$ $\tilde{x}_{M}(k, l) \mid$ is greater than any of $h_{1}$ or $h_{2}$, then $x(k, l)$ is of very small or of very large value with respect to its neighbouring pixels and most possibly a randomly-valued impulse. If the current pixel is an impulse, either constant or randomly-valued, it is excluded from the estimation of the median at the current and at any future window centered at $(k, l)$ yielding the modified median.

3. Calculation of the weighting coefficient $b(k, l)$.

This coefficient is given by the expression [1]:

$$
b(k, l)= \begin{cases}0, & \text { if } \alpha \sigma_{n}^{2} \geq \hat{\sigma}_{x}^{2} \\ \left(1-\alpha \frac{\sigma_{n}^{2}}{\hat{\sigma}_{x}^{2}}\right)^{\beta}, & \text { otherwise. }\end{cases}
$$

$\hat{\sigma}_{x}^{2}$ denotes the image variance estimated from the local "windowed" histogram by excluding the current pixel if it is detected as an impulse [1]. $\alpha$ and $\beta$ are appropriately chosen parameters in the interval $[0,1]$. The parameter $\alpha$ controls the threshold on the local $S N R$ up to which the high-frequency components are entirely suppressed. The parameter $\beta$ controls the suppression of noise close to edges.

4.Decision whether the current pixel belongs to a homogeneous region or to an edge.

The weighting factor $b(k, l)$ is compared to a predefined threshold $b_{t}$. If it is smaller than $b_{t}$, then the current pixel is assumed to belong to a homogeneous region. Otherwise, the current pixel belongs to an edge. The threshold $b_{t}$ lies in the interval $[0,1]$. Its selection is accomplished in accordance with the degree of corruption and the nature of noise. For highly corrupted images, its value is lower than 0.5 . If the image is corrupted by pure Gaussian noise of relatively medium variance, the threshold lies in the range $[0.65,0.85]$. A reliable method for the choice of the threshold $b_{t}$ is described in [1].

5.Novel window adaptation procedure. The proposed $M S A M$ differs from the $S A M$ filter in the window adaptation procedure used. $S A M$ employs isotropic filter windows of dimensions $3 \times$ 3 up to $11 \times 11$. In contrast to $S A M$, an anisotropic window adaptation procedure is proposed based on mathematical morphology erosions/ dilations with predefined structuring elements. Four structuring elements are employed, namely, $B_{1}, B_{2}, B_{3}$ and $B_{4}$ and their symmetric ones $B_{1}^{s}, B_{2}^{s}, B_{3}^{s}$ and $B_{4}^{s}$ illustrated in Fig. 1a. They are divided in even-angle SEs $\left(B_{1}, B_{2}, B_{1}^{s}, B_{2}^{s}\right)$ and in odd-angle SEs $\left(B_{3}, B_{4}, B_{3}^{s}, B_{4}^{s}\right)$. The window increment is performed by a dilation operation $W \oplus B_{i}$, where $W$ denotes the current filter window. The "direction" of increment depends on the choice of $B_{i}$. The result of the window growing $W \oplus B_{i}$ for an original $3 \times 3$ window size $W$ is shown in Fig. $1 \mathrm{~b}$. The thin dots belong to the original window $W$ while the bold dots denote the new pixels that have been appended to $W$ forming thus the new (larger) window. In an analogous fashion, the window decrement is performed by an erosion operation $W \ominus B_{i}$. This is demonstrated in Fig. $1 \mathrm{c}$ for $B_{1}, B_{1}^{s}, B_{2}, B_{2}^{s}$. The procedure of the window adaptation begins with a $3 \times 3$ square window and checks whether the central pixel belongs to an edge.

\section{If it does not belong to an edge:}

(a) An attempt is made to increase the window size by using the odd-angle SEs.

(i) If an edge is "hit" (e.g. $b(k, l)>b_{t}$ ) any odd-angle SE employed is excluded and the even-angle SEs that compose it are tested for possible window increment. For example, if $B_{4}$ is excluded, $B_{1}$ and $B_{2}$ are tested for possible window increment. This means that the sides of the mask are also separately checked expecting that an edge is possibly met at one side only. By doing so, maximal window increment is achieved.

(ii) If an edge is not met, then the odd-angle SE is used to increase the window size. The corresponding even-angle SEs are then excluded.

(b) In the next step, the odd-angle SEs, that have not been excluded in a previous step, are tested again. In the abovedescribed example, $B_{3}, B_{3}^{s}$ and $B_{4}^{s}$ remain to be tested. In other words, if it is known from a previous step that a window side meets an edge, this side is not considered again.

(c) The procedure continues until all the odd-angle and all the even-angle SEs are excluded or until at least one side reaches a maximal size (e.g., 11).

II. If the pixel belongs to an edge, the goal is to expand the mask in the neighbouring regions that are homogeneous. That is, the current pixel is labeled as a border pixel and the window increment is done towards the side of the edge where the pixel belongs to. To do so, the opposite side of the edge must be found and the increment of the filter window towards that direction must be prohibited. This is done as follows. The average value of the pixels on each of the four sides of a window of dimensions $3 \times 3$ is derived and the absolute difference between these average values and the current pixel is calculated. The side that corresponds to the greater difference is removed. The difference is a measure of deviation of the side pixels from the current one. The side that deviates the most is possibly the side that should be removed. The decrement of the initial window size is achieved by the operation of erosion with one of the SEs $B_{1}, B_{1}^{s}, B_{2}, B_{2}^{s}$ only. Subsequently, the window increases towards the remaining sides in the way described above by using appropriate SEs. For example, if erosion with $B_{1}$ were performed, $B_{1}, B_{2}, B_{2}^{s}, B_{3}, B_{4}^{s}$ would be used to increase 
further the window.

Finally, if the current pixel is detected as an impulse, the factor $b(k, l)$ is set to 0 (thus allowing maximum filtering).

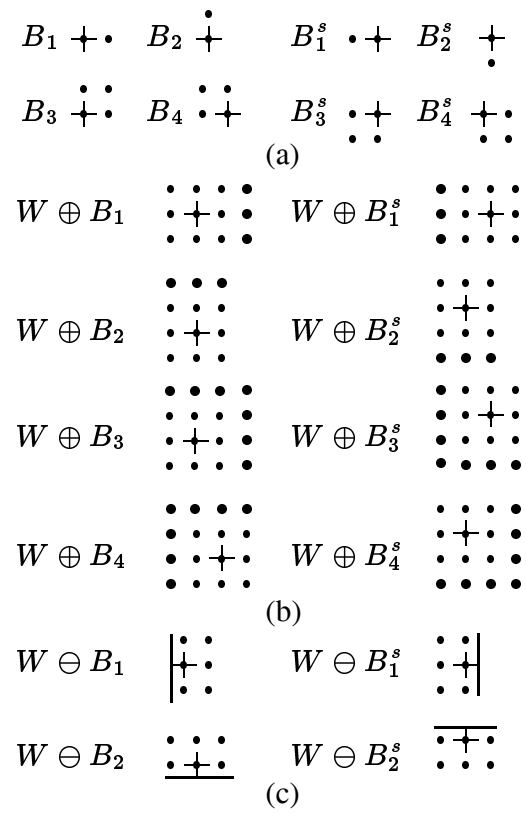

Figure 1: (a) Structuring Sets. (b) Window Increment using Dilation. (c) Window Decrement using Erosion.

\section{SIMULATION RESULTS}

The noise corruption process of [4] is adopted. White i.i.d. noise obeying the pdf of a Gaussian mixture given by:

$$
n \sim(1-\lambda) N\left(0, \sigma_{\nu}\right)+\lambda N\left(0, \frac{\sigma_{\nu}}{\lambda}\right)
$$

is added to the noise-free test images. Its mean value $E(n)$ is close to 0 while its variance is given by:

$$
\sigma_{n}^{2}=\sigma_{\nu}^{2}(1-\lambda+1 / \lambda)
$$

The contamination factor $\lambda$, along with the initial standard deviation $\sigma_{\nu}$, determine the degree of corruption. The result of this noise corruption process is a mixture of Gaussian and impulsive noise of varying characteristics. The special case of constant impulsive noise corruption is additionally examined in order to test the robustness of the proposed filter against this kind of noise.

Performance conclusions are drawn based on $S N R$ and $M A E$ values, as well as on the achieved visual quality of the filtered test images.

\subsection{Still Image Filtering}

The noise-free image "Airfield" has been used as test image in the still image filtering case. Part of the original image is shown in Fig. 2a. Three cases are examined. First, "Airfield" is corrupted by the contaminated noise of (9) using $\lambda=0.1$ and $\sigma_{\nu}^{2} \simeq 586.5$, which leads to severe corruption by both impulsive and Gaussian noise (Fig. 2b). Secondly, $\lambda$ is set equal to 1.0 which implies that only Gaussian noise is present in the noisy image. A value close

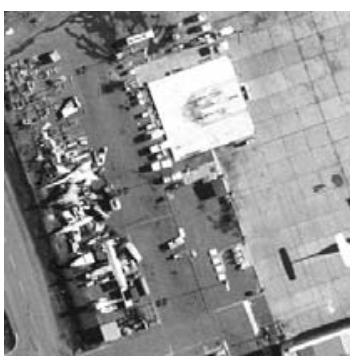

(a)

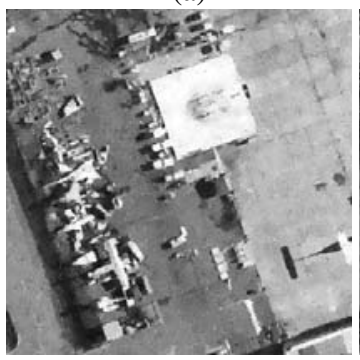

(c)

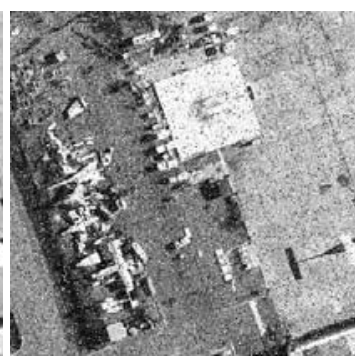

(b)

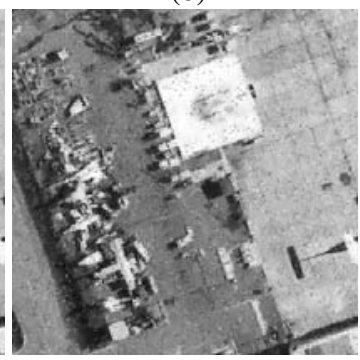

(d)
Figure 2: (a) Part of the noise-free image: Airfield. Same part (b) corrupted by contaminated noise, (c) processed by $M S A M$ and (d) processed by $S A M$.

to 474.0 has been used for $\sigma_{\nu}^{2}$. Finally, impulsive noise of $10 \%$ positive and $10 \%$ negative constant impulses has been added to "Airfield". The simulation results for the three cases are listed in Table 1. For comparison purposes, results obtained by using the $S A M$ and the median filter of dimensions $3 \times 3$ are also included in Table 1. Fig. 2c shows the output of the $M S A M$ filter cor-

\begin{tabular}{|c|c|c|c|c|c|c|}
\hline \multirow[t]{2}{*}{ Filter } & \multicolumn{2}{|c|}{ Contaminated } & \multicolumn{2}{|c|}{ Gaussian } & \multicolumn{2}{|c|}{ Impulsive } \\
\hline & $S N R$ & $M A E$ & $S N R$ & $M A E$ & $S N R$ & $M A E$ \\
\hline Noisy & 3.03 & 26.47 & 9.01 & 16.83 & -0.03 & 23.04 \\
\hline$M S A M$ & 11.72 & 11.00 & 12.80 & 10.18 & 14.47 & 4.60 \\
\hline$S A M$ & 9.27 & 13.16 & 12.46 & 10.76 & 13.41 & 3.08 \\
\hline Median & 11.18 & 12.27 & 12.38 & 10.65 & 12.58 & 7.69 \\
\hline
\end{tabular}

Table 1: Simulation Results: Still image case.

responding to the image part examined. The output of the $S A M$ filter is shown in Fig. 2d. The inspection of Table 1 manifests that the $M S A M$ filter outperforms the $S A M$ and the median filters in all cases. This is also verified by comparing Fig. $2 \mathrm{c}$ and $2 \mathrm{~d}$. Better impulse removal, edge preservation, edge noise filtering and Gaussian noise smoothing in flat regions are achieved.

\subsection{Image Sequence Filtering}

The "Mobile" image sequence has been used in the image sequence filtering experiments. Motivated by the success of motioncompensated (MC) filtering [5], motion compensation performed by block matching has been used to estimate the motion trajectory among the frames in the spatiotemporal filter window. Frame 4 was used as current frame, part of which is shown in Fig. 3a, Frame 0 as previous and Frame 8 as next.

Extension of the $M S A M$ filter structure to an MC-spatiotemporal counterpart involves the determination of the filter's spa- 


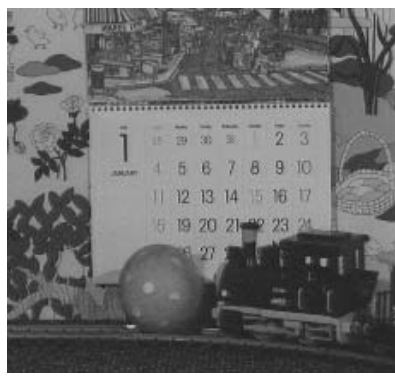

(a)

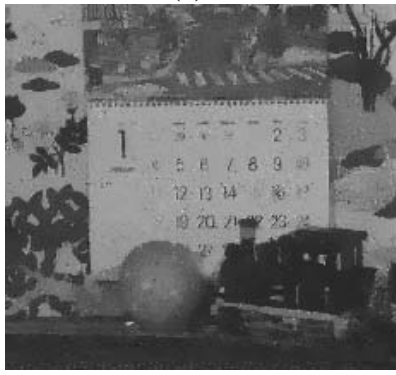

(c)

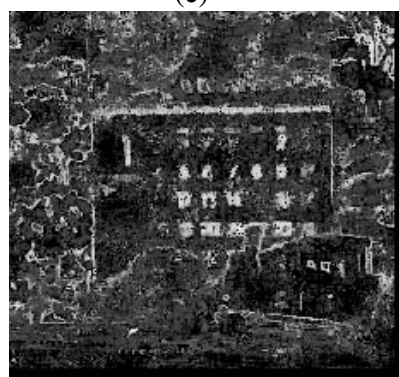

(e)

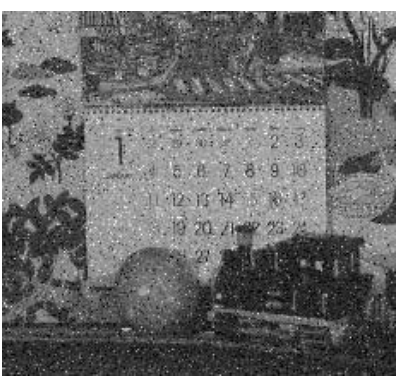

(b)

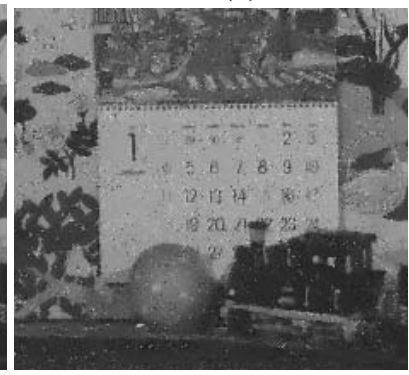

(d)

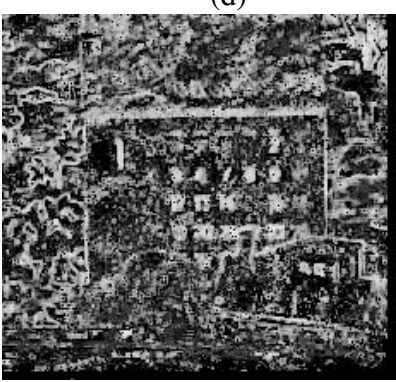

(f)
Figure 3: (a) Part of the noise-free Frame 4 of Mobile sequence. Same part (b) corrupted by contaminated noise, and processed by (b) MC $M S A M$ and (d) MC $S A M$. Edge detection achieved by: (e) MC $M S A M$ and (f) MC $S A M$.

tiotemporal window. This is composed by asymmetric spatial windows of variable dimensions defined with reference to the pixels on the motion trajectory. The spatial windows are adapted separately in each frame in the same way as the spatial $M S A M$ filter does.

The same noise cases are examined here as well. Frames 04-8 are corrupted by contaminated noise (9) using $\lambda=0.2$ and $\sigma_{\nu}^{2}=100.0$ (part of the corrupted Frame 4 is shown in Fig. 3b), Gaussian noise using $\lambda=1.0$ and $\sigma_{\nu}^{2}=225.0$ and constant impulsive noise with $7.5 \%$ positive and $7.5 \%$ negative impulses. The performance of the MC-spatiotemporal $M S A M$ filter is compared to the MC spatiotemporal $S A M$ filter (the structure of which is extended in a similar way to the $M S A M$ filter with the exception that symmetric spatial windows are used) and median filter of dimensions $3 \times 3 \times 3$. The simulation results are listed in Table 2, which shows that the MC $M S A M$ filter outperforms the other two in all cases. This is also proved by judging from the visual quality of the processed Frame by the MC $M S A M$ and the MC $S A M$ filters (Figs. $3 \mathrm{c}$ and $3 \mathrm{~d}$ respectively). Figs. $3 \mathrm{e}$ and $3 \mathrm{f}$ illustrate the value of $b(k, l)$ at each pixel appropriately scaled. A value close
Table 2: Simulation Results: Image Sequence case.

\begin{tabular}{|c|c|c|c|c|c|c|}
\hline \multirow[t]{2}{*}{ Filter } & \multicolumn{2}{|c|}{ Contaminated } & \multicolumn{2}{|c|}{ Gaussian } & \multicolumn{2}{|c|}{ Impulsive } \\
\hline & $S N R$ & $M A E$ & $S N R$ & $M A E$ & $S N R$ & $M A E$ \\
\hline Noisy & 4.97 & 14.04 & 8.68 & 11.95 & -1.89 & 17.82 \\
\hline$M S A M$ & 11.17 & 7.27 & 13.36 & 6.20 & 15.74 & 2.31 \\
\hline$S A M$ & 10.16 & 7.67 & 13.15 & 6.33 & 15.37 & 1.61 \\
\hline Median & 10.03 & 8.42 & 10.00 & 8.75 & 10.10 & 7.20 \\
\hline
\end{tabular}

to 1.0 indicates edge region while a value close to 0.0 a flat region. It is seen that the $S A M$ edge detection produces thicker edges which implies less filtering in those areas. Furthermore, pixels in flat regions are seen to be mistakenly assigned as edge pixels by the $S A M$ filter due to its reduced impulse detection capabilities. Thus, the MC $M S A M$ filter achieves better impulse removal and noise smoothing.

\section{CONCLUSIONS}

The performance of the $M S A M$ filter proves to be very satisfactory in both still images and image sequences. Especially at high corruption levels, its performance significantly surpasses that of the other filters under study. This is attributed to three factors: (i) the use of large window sizes at flat regions, (ii) the anisotropic window adaptation that allows a higher noise suppression close to edges, either spatial or temporal, and (iii) the improved impulse detection mechanism. Such good performance is obtained without any reference image or training process and in a single pass. Furthermore, the poor performance of median filters in presence of Gaussian noise is diminished. Moreover, the $M S A M$ filter achieves a noticeable improvement in the visual perception of the filtered images/frames. Edges are preserved at a high extent, while noise is better suppressed in flat regions and impulses are more efficiently removed.

However, a minor disadvantage is its inability to preserve very small details and thin lines. Furthermore, the anisotropic window adaptation process requires more computational effort due to erosion and dilation computations.

\section{REFERENCES}

[1] R. Bernstein, "Adaptive Nonlinear Filters for Simultaneous Removal of Different Kinds of Noise in Images", IEEE Trans. on CAS, vol. 34, pp. 1275-1291, Nov. 1987.

[2] R. Sucher, "A Recursive Nonlinear Filter for the Removal of Impulse Noise", in Proc. of ICIP'95, pp. 183-186, 1995.

[3] M. Lightstone and E. Abreu and S.K. Mitra and K. Arakawa, "A New Filtering Approach for the Removal of Impulse Noise from Highly Corrupted Images", IEEE Trans. on IP, vol. 5, no. 6, pp. 1012-1025, June 1996.

[4] M. Gabbouj and I. Tabus, "TUT noisy image database", Technical Report ISBN 951-722-281-5, Signal Proc. Lab, Tampere Univ. of Technology, Tampere, Finland, Dec. 1994.

[5] J.C. Brailean, R.P. Kleihorst, S. Efstratiadis, A.K. Katsaggelos, R.L. Lagendijk, "Noise Reduction Filters for Dynamic Image Sequences: A Review", Proc. of the IEEE, vol. 83, no. 9, pp. 1272-1292, Sep. 1995. 